\title{
Depression, Sexual Functioning and the Life Satisfaction of Pakistani Women with and without Polycystic Ovary Syndrome
}

\author{
Muneeba Shakil ( $\square$ muneebashakeel@cuilahore.edu.pk) \\ COMSATS University Islamabad - Lahore Campus https://orcid.org/0000-0002-7706-5211 \\ Amina Wajid \\ COMSATS University Islamabad - Lahore Campus \\ Farzana Ashraf \\ COMSATS University Islamabad - Lahore Campus
}

\section{Research article}

Keywords: polycystic ovarian syndrome, depression, sexual functioning, life satisfaction, MOANOVA, demographic characteristics

Posted Date: February 10th, 2020

DOI: https://doi.org/10.21203/rs.2.23054/v1

License: (c) (i) This work is licensed under a Creative Commons Attribution 4.0 International License. Read Full License 


\section{Abstract}

Background: One hormonal disorder, polycystic ovary syndrome (PCOS) has been frequently diagnosed in Pakistani women of reproductive age in the last few years. This study was carried out to determine whether there were any differences in the levels of depression, sexual function and/or life satisfaction of Pakistani women with and without PCOS related to their demographic situation. It was hypothesized that significant differences would be identified.

Method: A sample of 126 women of reproductive age between $18-45$ years [63 diagnosed with PCOS by their gynaecologist (Mage = $27.86, S D=4.58$ ) and 63 not suffering from any hormonal or reproductive issues (Mage = 27.89, SD = 3.92)] was selected through purposive and convenient sampling techniques respectively from Lahore, Pakistan. The participants responded to a Self-Developed Demographic Information Form, Siddiqui Shah Depression (SSDS), Life Satisfaction Scale (LS) and Female Sexual Functioning Index (FSFI).

Results: A cross sectional study design with $2 \times 3$ (age $\times$ Education), MANOVA was carried out and the analysis revealed significant differences between the variables in relation to the demographic situation of the sample women with/without PCOS.

Conclusion: This research adds to Pakistan's health psychology literature on the phenomena researched. Future research endeavours in this understudied research area are also suggested.

\section{Background}

The hormonal disorders polycystic ovary syndrome (PCOS) is very common and has been frequently diagnosed in Pakistani women of reproductive age for the last few years. Women with this condition have prolonged, irregular or painful menstruation cycles along with increased androgen levels. Polycystic ovaries mostly develop a number of small collections of fluid i.e. follicles which can result in enlarged ovaries, reducing their ability to release eggs, leading to fertility issues. The signs of PCOS develop with the first menstrual cycle during puberty and usually stay undetected. However, other symptoms develop after weight gain [1].

The exact aetiology of this disorder is not yet known; however, its treatment along with weight reduction is said to limit the risk of longterm complications, including diabetes and heart disease. The factor that might contribute most to the development of this hormonal disorder is said to be an Excessive level of insulin produced by the pancreas, allowing the cells to use body's primary energy supply. Excess insulin increases the production of androgen, causing difficulty in ovulation. Low grade inflammation results from the polycystic ovaries producing more androgen because of a lack of white blood cells to fight infection, which can lead to heart problems as well. The disorder's complications include diabetes, infertility issues, miscarriages, premature deliveries, high blood pressure, increased cholesterol level, cardiovascular disease, sleep disturbances, uterine cancer, depression, anxiety and eating disorders [1].

The western literature suggests that PCOS is associated with many physical and psychological changes in women which affect their psychological functioning and life satisfaction in general. The findings suggest that there are significant differences between women with and without PCOS with respect to depression and satisfaction with life. However, no significant difference has been found relating to their demographic characteristics $[2,3,4]$. Although PCOS is a common disorder, not much is known about its effects on sexual function, the sexual response cycle and satisfaction. One study found sexual dysfunction present in $27.2 \%$ of women with PCOS but only in $24.4 \%$ of women without PCOS, and the former group's increased BMI was also associated with a reduction in their orgasms [5].

A study on depression and anxiety in Pakistani women suffering from PCOS found that $42 \%$ suffered from anxiety, and $20 \%$ had both depression and anxiety compared to the control group, where $11 \%$ women suffered from anxiety, and $3.5 \%$ had both depression and anxiety [6]. Regarding Pakistani Urban Women's awareness of PCO symptoms, 36.7\% were aware of symptoms related to hirsutism; $19.5 \%$ were aware their facial hair symptom, $6.5 \%$ had Breast hair and $6.5 \%$ were aware of other forms of hirsutism [7]. Mezaal, Nader, and Aziz in 2015 [8] reported that increased insulin production in PCOS women is indirectly involved in the production of sex hormones. Insulin resistance in these women affects their sexual functioning adversely, which in turn can lead to the development of PCOS. Amiri, Tehrani, Esmailzadeh, Tohidi, Azizi and Basirat in 2018 [9] calculated the relationship between PCOS and Sexual function and found that $45.75 \%$ of the PCOS women met the criteria for sexual dysfunction. The literature suggests that the physical, medical and psychological symptoms of PCOS significantly affect the quality of life and satisfaction with life of such women $[10,11,12,13]$. 
The literature includes cross-sectional studies of PCOS and Non-PCOS women with depression, anxiety, life satisfaction and sexual function. However, there is gap in knowledge with respect to the demographic characteristics and differences in depression, sexual functioning and life satisfaction of women suffering from PICOS and those not suffering from PCOS. This phenomenon is understudied in Pakistan with reference to these multiple aspects, and research is needed focusing on this health-related issue with a number of psychological variables. The present study was carried out with the objective of determining differences in the level of depression, sexual functioning and life satisfaction of Pakistani women with and without (Control Group) polycystic ovary Syndrome (PCOS). A further aim was to identify any differences related to the participants' (a) age and (b) education. It was hypothesized that "there would be significant differences in the aforementioned variables of women with and without PCOS in relation to their demographic characteristics".

\section{Methods}

\section{Study Design and settings}

A cross-sectional study design $2 \times 3$ (age $\times$ education level) was used to discover any differences in depression, sexual functioning and life satisfaction related to the demographics of the female participants with and without polycystic ovarian syndrome. Data were collected from Mayo hospital, Services, Sir Ganga Ram, Gulab Devi, and combined military hospital of Pakistan, Lahore. These hospitals have gynaecology departments with a large number of females diagnosed with polycystic ovary syndrome.

\section{Study Population, Sampling And Inclusion Criteria}

A sample of 63 female of reproductive age $18-45$ (Mage $=27.86, S D=4.58)$ years, diagnosed with Polycystic Ovarian Syndrome by their gynaecologist were selected through purposive and snowball sampling. A sample of 63 women of reproductive age 18-45 (Mage $=27.89, S D=3.92$ ) years not suffering from any hormonal or reproductive issues (Control Group) were selected through convenience sampling. The demographic characteristics of the participants are summarized in Table 1.

Women suffering from polycystic ovarian syndrome (PCOS) were only included in the study if they: (1) were married; (2) in the reproductive age group; (3) had been diagnosed by a gynaecologist; (4) were undertaking treatment; (5) were living with their husbands and (6) only resided in Pakistan. They were excluded from participation in the study if they: (1) suffered from a mental illness; and (2) had any other physical illness. Women in the control group were included in the study only if they were: (1) married; (2) in the reproductive age group; (3) living with their husbands and (6) residing only in Pakistan. They were excluded from the study if they: (1) had been diagnosed with a mental illness; and (2) had not had children.

\section{Data Collection Tools}

\section{Informed Consent Form}

The purpose of the study and request for the participants' voluntary participation was outlined in the informed consent form. The consent form included a written agreement to participate in the study.

\section{Demographic Information Form}

The form included basic information about the participants including their age, academic qualifications, birth order, socioeconomic status, years of marriage, family setup, number of children, number of siblings, residential area and the monthly income of the family (see Table 1).

\section{The Siddiqui Shah Depression Scale}

This is a 36 items culturally appropriate scale for measuring depression developed by Siddique Shah in 1997 [14] in Urdu. The scale contains 12 items of mild depression, 12 for moderate and 12 for severe depression to measure both clinical and non-clinical

Page $3 / 9$ 
depression. The scale ranges from $0=$ never, to $3=$ every time. The split half reliability of SSDS is $(r=0.79)$ and $(r=0.84)$ for clinical and $(r=0.80)$ and $(r=0.89)$ for non-clinical samples respectively, and the alpha coefficients for the clinical and non-clinical samples are 0.91 and 0.89 .

\section{Female Sexual Function Index (FFSI) [15]}

The Female Sexual Function Index (FSFI) is a self-reporting measure of for sexual functioning. It has been validated for patients diagnosed with disorders of sexual arousal. It is a published scale which has been validated and normed on the sample of women clinically diagnosed with female sexual dysfunction. The range of Cronbach's a coefficients is 0.841 to 0.970 and for five factors (desire, arousal, lubrication, orgasm, satisfaction and pain) face validity is 7. 556, 3.457, 2.939, 2.926 and .633 respectively. The scale can discriminate between women experiencing any sexual arousal disorder and those who are normal, without any arousal difficulty in 5 domains: desire, arousal, lubrication, orgasm, satisfaction, and pain [16]. The FSFI was translated into Urdu by Rehman, Mahmood, Sheikh; Sultan; and Khan, 2015 [17], which is the version used in the study.

\section{Life Satisfaction Subscale}

The subjective well-being scale is a valid and reliable of measure of subjective wellbeing based on three subscales: five items in the Life Satisfaction subscale (LS), 12 items for the Positive Affect (PA) and 12 items for the Negative Affect (NA) subscales, developed by Fazeela Moghal in 2012 [18]. The life satisfaction subscale was used in the study to assess the life dissatisfaction of women with polycystic ovarian syndrome. Cronbach's alpha values for the three subscales is $.83, .84$ and .81 respectively.

\section{Procedure}

First, a brief introduction to the research was given and permission granted by the heads of departments of the hospitals concerned with patients diagnosed with PCOS. The PCOS ample was selected through purposive and snow ball sampling from different private and government hospitals in Lahore. Married females without PCOS were approached in person from Lahore, Pakistan through convenient sampling. The informed consent form was explained to the participants and their verbal as well as written consent to voluntarily participate in the study was obtained. They answered the Demographic Information Sheet; Siddiqui Shah Depression Scale; Female Sexual Functioning Index; and Life Satisfaction Scale questions after they had been informed of the purpose of the study. Finally, the information sheets from both groups were collected and the appropriate statistics applied and the results calculated.

\section{Data Analysis}

Based on the examination of the demographic characteristics, the variables were grouped to form categories. Descriptive statistics, including the mean, standard deviations, frequencies and percentages as well as inferential statistics through the multivariate analysis of variance (MANOVA) were used to determine the mean differences in depression, sexual function and life satisfaction of the female participants with and without polycystic ovarian syndrome with respect to their demographics. IBM SPSS Statistics 24 was used to carry out the analysis.

\section{Results}

\section{Descriptive Statistics}

Table 1

Frequencies of Demographic Characteristics of women with and without PCOS 


\begin{tabular}{|lllll|}
\hline Variables & \multicolumn{3}{l}{ Women with PCOS } & \multicolumn{2}{l|}{ Women without PCOS } \\
\hline & $\mathrm{F}$ & $\%$ & $\mathrm{f}$ & $\%$ \\
\hline Education & & & & \\
\hline Metric & 0 & 0 & 3 & 4.8 \\
\hline Inter & 3 & 4.8 & 3 & 4.8 \\
\hline Graduation & 31 & 49.2 & 36 & 57.1 \\
\hline Masters & 29 & 46.0 & 21 & 33.3 \\
\hline Family System & & & & \\
\hline Nuclear & 23 & 36.5 & 19 & 30.2 \\
\hline Joint & 40 & 63.5 & 44 & 69.8 \\
\hline Work Status & & & & \\
\hline Working & 21 & 33.3 & 17 & 27 \\
\hline Non-Working & 42 & 66.7 & 46 & 73 \\
\hline Socioeconomic Status & & & & \\
\hline Lower & 10 & 15.9 & 4 & 6.3 \\
\hline Middle & 33 & 52.4 & 43 & 68.3 \\
\hline Upper & 20 & 31.7 & 16 & 25.4 \\
\hline
\end{tabular}

The data was analyzed by using $2 \times 2$ (age $\times$ Education level), multiple analysis of variance (MANOVA). Table 1 (above) represents the distribution of sample across age $\times$ Education level.

Table 2 show that the mean differences in depression, life satisfaction and sexual function according to the participants' demographic characteristics were significant. Interpreting the findings revealed that the women with PCOS suffered more depression, impaired sexual function and had less satisfaction with life compared to the control group. However, interesting there were findings with reference to the participants' age, revealing that middle aged women with PCOS suffered less depression, had better sexual function and satisfaction with life compared to young PCOS sufferers.

Table 3 show an interactive effect analysis of age and education which was quite interesting in terms of the findings; lower educated women with PCOS were found to have less depression - the level of depression increased along with their level of education. On the other hand, the women in the control group suffered less depression as compared to the lower educated women in the control more educated group. In addition, the life satisfaction of the lower educated PCOS women was better than that of the lower educated control group.

Table 2

Mean main effect differences of depression, sexual function and life satisfaction across age groups and education levels between women with and without PCOS.

\section{Main Effect}




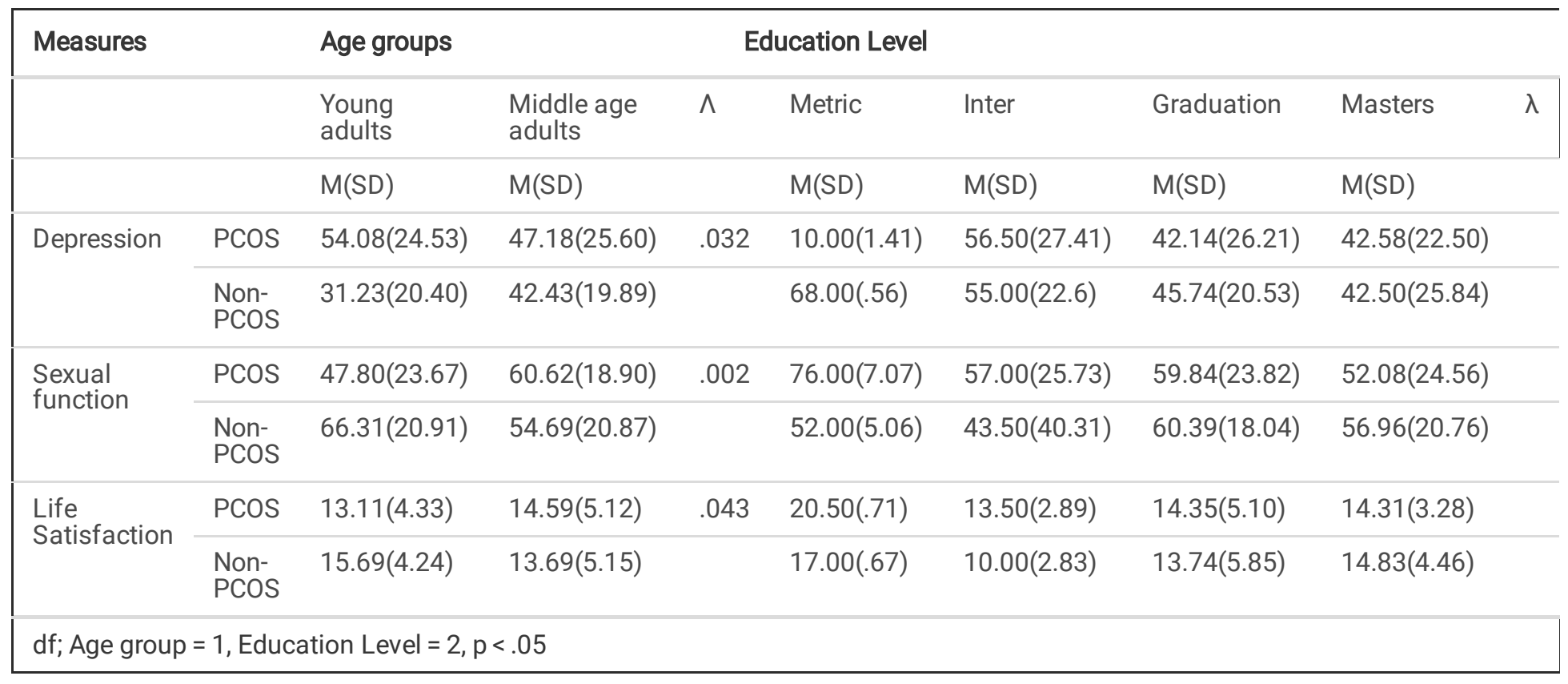

Table 3

Mean interactive effect differences of depression, sexual function and life satisfaction across age groups and education levels between women with and without PCOS.

\section{Interactive Effect}

\begin{tabular}{|c|c|c|c|c|c|c|c|c|}
\hline \multicolumn{2}{|l|}{ Measures } & \multicolumn{2}{|l|}{ Age groups } & \multicolumn{3}{|c|}{ Education Level } & \multirow[b]{2}{*}{ Masters } & \multirow[b]{2}{*}{$\lambda$} \\
\hline & & $\begin{array}{l}\text { Young } \\
\text { adults }\end{array}$ & $\begin{array}{l}\text { Middle age } \\
\text { adults }\end{array}$ & Metric & Inter & Graduation & & \\
\hline & & $M(S D)$ & $M(S D)$ & $M(S D)$ & $M(S D)$ & $M(S D)$ & $M(S D)$ & \\
\hline \multirow[t]{2}{*}{ Depression } & PCOS & $54.08(24.53)$ & $47.18(25.60)$ & $10.00(1.41)$ & $56.50(27.41)$ & $42.14(26.21)$ & $42.58(22.50)$ & .000 \\
\hline & $\begin{array}{l}\text { Non- } \\
\text { PCOS }\end{array}$ & $31.23(20.40)$ & 42.43(19.89) & $68.00(.56)$ & $55.00(22.6)$ & $45.74(20.53)$ & $42.50(25.84)$ & \\
\hline \multirow{2}{*}{$\begin{array}{l}\text { Sexual } \\
\text { functioning }\end{array}$} & PCOS & $47.80(23.67)$ & $60.62(18.90)$ & $76.00(7.07)$ & $57.00(25.73)$ & $59.84(23.82)$ & $52.08(24.56)$ & .000 \\
\hline & $\begin{array}{l}\text { Non- } \\
\text { PCOS }\end{array}$ & 66.31(20.91) & $54.69(20.87)$ & $52.00(5.06)$ & $43.50(40.31)$ & $60.39(18.04)$ & $56.96(20.76)$ & \\
\hline \multirow{2}{*}{$\begin{array}{l}\text { Life } \\
\text { Satisfaction }\end{array}$} & PCOS & $13.11(4.33)$ & $14.59(5.12)$ & $20.50(.71)$ & $13.50(2.89)$ & $14.35(5.10)$ & $14.31(3.28)$ & .000 \\
\hline & $\begin{array}{l}\text { Non- } \\
\text { PCOS }\end{array}$ & $15.69(4.24)$ & $13.69(5.15)$ & $17.00(.67)$ & $10.00(2.83)$ & $13.74(5.85)$ & $14.83(4.46)$ & \\
\hline
\end{tabular}

\section{Discussion}

The main effect mean differences between the variables with respect to the participants' age revealed that the women in the PCOS group experienced more depression than the control group. The sexual function of young PCOS diagnosed women was more impaired compared to the control group. However, middle aged PCOS women had comparatively better sexual function than young PCOS women. Women with PCOS were less satisfied with life than the control group. However, the middle aged PCOS women had comparatively more satisfaction with life than the young PCOS women. 
The results of this study with respect to depression, sexual function and life satisfaction are consistent with the literature. However, adding to the existing literature is the role of age in the sexual function of women with PCOS. The study findings have revealed that middle aged PCOS women had much better sexual function, which might be because of the reduction in androgen production because of the decreased activity of adrenal gland and ovarian aging. The literature suggests that the volume of ovaries and number of follicles also decreases with age. These age-related changes are said to reduce the complications of

PCOS. These changes might also have affected the participants' level of depression, which was found to be lower, and satisfaction with life, which was found to be better than in young PCOS women [19].

The interactive effect analysis of age and education produced quite interesting findings i.e. the lower educated women with PCOS were found to have less depression and depression levels increased with higher levels of education. On the other hand, the more educated women in the control group had less depression compared to the lower educated women in the control group. In addition, the life satisfaction of the lower educated PCOS diagnosed women was better than that of the lower educated control group. It is a well-established and much investigated notion that education increases awareness, which leads to better women in the coping skills, which in turn reduce the number of problems. Some of this study's findings are consistent with this concept. However, after determining the differences in depression levels of women with PCOS in relation to their age, the results revealed different findings from what seemed to be an established norm. The more educated women with PCOS suffered more from depression than the ones with less education. This effect might be for several reasons, one of which might be the ego function of highly educated females, who might not find it easy to accept that they have any medical condition leading to fertility issues. This non-acceptance of their situation might be due to having sufficient awareness of the condition and its frequent follow up treatments, which might have led to an increased level of depression in highly educated PCOS women. On the other hand, lack of awareness or ignorance of the symptoms in less educated women might have led to their lower levels of depression and better satisfaction with life. Ignorance is bliss, in some situations, not knowing much about a problem can cause an individual to accept the illusion that there is no problem or it is one that will get better with time.

The results further revealed that the highly educated PCOS women had better sexual function compared to the lower educated PCOS women. Interestingly, the sexual function of the more educated PCOS women was better than that of the more educated women in the control group. However, the sexual function of the lower educated control group of women was better than that of the lower educated PCOS women. As mentioned, the findings in the literature to date did suggest that the sexual function of the control group would be better than the sexual function of the women with PCOS, and the results in this research concur with that finding. The more educated women, due to their awareness of sexual satisfaction and of their sexual response cycle, may pay more attention to improving their performance during sexual activity. Talking about sex or being aware of it is considered a taboo topic in Pakistan, consequently, without counselling, a number of sexual problems persist in Pakistani women. The chances of this problem increase even more if the women concerned are uneducated or less educated. For them, sexual activity is just an obligation in order to bear a child and fulfil their responsibility as a female. Lack of awareness of sexual satisfaction can lead to poor performance on the part of both partners. Even under the best circumstances, most couples in Pakistan find it difficult to talk about sex. Because well-educated women tend to be more confident, aware and good at communication, this serves as a cornerstone to help them develop a healthy sexual relationship with their partners. Establishing a dialogue is the best way to have a better sex life and closer emotional bonding; better education might be a supportive force contributing indirectly to better sexual function.

\section{Conclusion}

This research is an addition to Pakistan's health psychology literature with reference to the discussed phenomena. The dearth of studies specific to south Asian culture means it makes a significant contribution to the gap in knowledge. The findings of the present study also offer insights into the demographically related factors which can influence feelings of depression, life satisfaction and the sexual function of women suffering from this commonly diagnosed endocrine hormonal disorder. Health psychologists would be better able to offer support if the gaps in knowledge with respect to several demographic characteristics of women with PCOS could filled by studying to their psychological variables.

\section{Limitations And Future Suggestions}


In conducting a study, the aim is to construct it in best possible way; however, after starting it, different barriers may arise which force deviation from the ideal path. Our study constraints included limited self-financial resources, which meant we were unable to gather more data through probability sampling from women suffering from polycystic ovary syndrome all over Pakistan. For a future endeavour, research taking a larger representative sample of the target population from other provinces of Pakistan while, investigating other demographic and psychological variables would be very valuable.

\section{Declarations}

\section{Ethics approval and consent to participate}

This study was conducted after being approved by the Approval and Ethical Committee of Department of Humanities, COMSATS University Islamabad, Lahore Campus. Besides this, permission was also taken from the head of genecology departments of concerned hospitals. Their written permission for voluntary participation in the study was also taken.

\section{Consent for publication}

Consent for publication is not applicable in this study as participants confidentiality has been maintained.

\section{Availability of data and materials}

The datasets used and/or analysed during the current study are available from the corresponding author on reasonable request.

\section{Competing Interests}

The authors declare there are no competing interests.

\section{Funding}

The authors state that no external funding was available

\section{Authors Contribution}

All authors made substantive intellectual contributions to this study to qualify as authors.

Dr. Muneeba Shakil conceived the idea, designed the study and performed statistical analysis. An initial draft of the manuscript was also written by her. Ms. Amina Wajid collected "data."

Dr. Farzana Ashraf re-drafted parts of the manuscript, helped with confirmatory factor analysis and provided helpful advice on the final revision of draft. All authors were involved in writing the manuscript. All authors have read and approved the final manuscript.

\section{Acknowledgement}

We acknowledge the voluntary participation of our respondents in this study

\section{References}

1. Mayo Clinic. Polycystic ovary syndrome (PCOS). [https://www.mayoclinic.org/diseases-conditions/pcos/symptoms-causes/syc20353439]. Accessed 23 Oct 2019.

2. Ferriday BD, Guenther N, Strauss B, Balen AH, Dye L. Quality of life and psychological wellbeing in polycystic ovary syndrome. Hum Reprod. 2007; 8:2279-2286. https:// doi:10.1093/humrep/dem108.

3. Borghi L, Leone D, Vegni E, Galiano V, Lepadatu C, Sulpizio P, Garzia E. Psychological distress, anger and quality of life in polycystic ovary syndrome: associations with biochemical, phenotypical and socio-demographic factors. J Psychosom Obstet Gynaecol. 2018; 39 (2). https://doi.org/10.1080/0167482X.2017.1311319.

4. Toth E. Depression, anxiety and satisfaction with life in patients with polycystic ovary syndrome. Endocrine and Metabolic Syndrome. Proceedings of the 3rd World Congress on Polycystic Ovarian Syndrome. 2017; p15-17. San Antonio, USA 
https://www.longdom.org/proceedings/depression-anxiety-and-satisfaction-with-life-in-patients-with-polycystic-ovary-syndrome19057.html. Accessed 24 Oct 2019.

5. Dale W, Stovall JL, Scriver AH, Clayton, Williams CD, Pastore LM. Sexual Function in Women with Polycystic Ovary Syndrome. J Sex Med. 2012; 9(1): 224-230. https:// doi: 10.1111/j.1743-6109.2011.02539.x.

6. Zehra S, Arif, A, Anjum N, Azhar A, Qureshi MA. Depression and anxiety in women with polycystic ovary syndrome from Pakistan. Life Sci J.2015; 12(3):1-4. http://www.lifesciencesite.com. Accessed 24 Oct 2019.

7. Gul S, Zahid SA, Ansari A. PCOS: Symptoms and Awareness in Urban Pakistani Women. Int J PharmaRes HealthSci. 2014; 2 (5): 356-360. https://www.pharmahealthsciences.net. Accessed 25 Oct 2019.

8. Mezaal MI, Nader MI, Aziz H. Molecular study of insulin resistance and polycystic ovary syndrome. Am J Med Sci 5. 2019; 3: $121-125$.

9. Amiri FN, TehraniFR, Esmailzadeh S, Tohidi M, Azizi F, Basirat Z. Sexual function in women with polycystic ovary syndrome and their hormonal and clinical correlations. Int J Impot Res. 2018; 30: 54 61. https://www.nature.com/articles/s41443-017-0006-2. Accessed 25 Oct 2019.

10. De Leo V, Musacchio MC, Cappelli V, Massaro MG, Morgante G, Petraglia F. Genetic hormonal and metabolic aspects of PCOS: An update. REPROD BIOL ENDOCRIN. 2016; 14:38. https:// doi: 10.1002/sm2.77.

11. Khomami MB, Tehrani FR, Hashemi S, Farahmand M, Azizi F. Of PCOS symptoms, hirsutism has the most significant impact on the quality of life of Iranian women. PLoS ONE 2015; 10(4). https://doi.org/10.1371/journal.pone.0123608.

12. Martin ML, Halling K, Eek D, Krohe M, Paty J. Understanding polycystic ovary syndrome from the patient perspective: A concept elicitation patient interview study. Health Qual Life Outcomes. 2017; 15:162. https://hqlo.biomedcentral.com/articles/10.1186/s12955-017-0736-3. Accessed 26 Oct 2019.

13. Taghavi SA, Bazarganipour F, Hugh-Jones S, Hosseini N. Health-related quality of life in Iranian women with polycystic ovary syndrome: A qualitative study. BMC Womens Health 15. 2015. https://bmcwomenshealth.biomedcentral.com/articles/10.1186/s12905-015-0272-4. Accessed 26 Oct 2019.

14. Shah, S. Manual of Depression Scale. National Institute of Psychology, Quaid e Azam University, Islamabad. 1992; 1-39.

15. Function Index (FSFI): Translation, Validation, and Cross-Cultural Adaptation of an Urdu Version "FSFI-U". J Sex Med. 2012; 3(4): 244-250. https://doi.org/10.1111/j.1743-6109.2012.02714.x.

16. Meston CM. Validation of the Female Sexual Function Index (FSFI) in Women with Female Orgasmic Disorder and in Women with Hypoactive Sexual Desire Disorder. J Sex Marital Ther. 2003; 29(1): 39-46. https://doi: 10.1080/713847100.

17. Rehman K, Mahmood MA, Sheikh SS, Sultan T, Khan, MA. The Female Sexual Function Index (FSFI): Translation, Validation, and Cross-Cultural Adaptation of an Urdu Version "FSFI-U". Sex Med. 2015; 3(4):244-50. doi: 10.1002/sm2.77.

18. Moghal, F. Correlates of Subjective Well-Being Measured by Indigenously Developed and Validated ICP Subjective Well-Being Scale (SWBS).2012. Unpublished Doctoral Dissertation. Institute of Clinical Psychology, University of Karachi. Karachi-Pakistan.

19. Hsu MI. Changes in the PCOS phenotype with age. Steroids. 2013; 78(8):761-6. https://doi: 10.1016/j.steroids.2013.04.005. 